\title{
Optimizing Adjuvant Radiation Planning Outcomes in Patients with Synchronous Bilateral Breast Cancer
}

\author{
Mohamed M. Alhefny ${ }^{1}$, Hany S. Attallah², Mahmoud Abdallah'2, Adel Yassin ${ }^{3}$, \\ Khaled M. El-Shahat ${ }^{3}$, Ahmed A. Obaya ${ }^{4}$ \\ ${ }^{1}$ Department of Clinical Oncology, Faculty of Medicine, Ain Shams University, Cairo, Egypt \\ ${ }^{2}$ Department of Radiation Oncology, International Medical Center, Cairo, Egypt \\ ${ }^{3}$ Department of Clinical Oncology, Faculty of Medicine, Al-Azhar University, Cairo, Egypt \\ ${ }^{4}$ Department of Clinical Oncology, Faculty of Medicine, Zagazig University, Zagazig, Egypt \\ Email: *Alhefny@med.asu.edu.eg
}

How to cite this paper: Alhefny, M.M., Attallah, H.S., Abdallah, M., Yassin, A., El-Shahat, K.M. and Obaya, A.A. (2021) Optimizing Adjuvant Radiation Planning Outcomes in Patients with Synchronous Bilateral Breast Cancer. Advances in Breast Cancer Research, 10, 110-119.

https://doi.org/10.4236/abcr.2021.103009

Received: June 28, 2021

Accepted: July 23, 2021

Published: July 26, 2021

Copyright $\odot 2021$ by author(s) and Scientific Research Publishing Inc. This work is licensed under the Creative Commons Attribution International License (CC BY 4.0).

http://creativecommons.org/licenses/by/4.0/ (c) (i) Open Access

\begin{abstract}
Background: Breast cancer is the most common cancer diagnosed worldwide, synchronous bilateral breast cancer accounts for unique entity of the disease, particularly post-operative radiotherapy for Synchronous Bilateral Breast Cancer (SBBC) is challenging with lack of evidence about the best irradiation technique. In this study, we tried to explore the optimum radiotherapy technique regarding the dosimetric parameters. Methods: We recruited 15 SBBC patients in whom post-operative radiotherapy was indicated and we established three plans for each patient using 3DCRT, IMRT and VMAT, and then we compared the three plans as regard target volume coverage parameters and organs at risk (OAR) doses. Results: We found that PTV coverage parameter was superior with IMRT compared with 3DCRT and VMAT in terms of Dmean $(\mathrm{p}=0.001)$, $\mathrm{D} 95 \%(\mathrm{p}=0.001), \mathrm{D}_{\max }(\mathrm{p}=0.0001)$, conformity index $(\mathrm{p}=0.0001)$ and $\mathrm{HI}(\mathrm{p}$ $=0.0001)$. Doses to OAR were not significantly different between the three techniques in cardiac dose and LAD maximum dose, but 3DCRT was superior in LAD mean dose $(\mathrm{p}=0.03)$ and lung volume receiving $20 \mathrm{~Gy}(\mathrm{~V} 20)$ and 10 Gy $(\mathrm{V} 10)(\mathrm{p}=0.0001)$, but this difference was non-significant between 3DCRT and IMRT ( $\mathrm{p}=0.4$ and 0.06 respectively), while VMAT led to the highest doses to LAD and lung. Conclusions: IMRT showed the best target coverage parameters in post-operative radiotherapy for SBBC compared with 3DCRT and VMAT. For OAR doses IMRT showed comparable results with 3DCRT, while VMAT delivered a significantly higher dose to OAR.
\end{abstract}

\section{Keywords}

Synchronous Bilateral Breast Cancer (SBBC), 3DCRT, IMRT, VMAT, Target 
Volume Coverage, OAR

\section{Introduction}

Breast cancer is the most common cancer in women, synchronous bilateral breast cancer (SBBC) accounts for about $1 \%-2 \%$ of breast cancer patients [1]. Despite low incidence, it represents significantly shorter overall survival than that of unilateral breast cancer [2]. There is lacking of standard guideline for treating SBBC. And owing to the increasing patient preference for breast-conserving treatment, synchronous bilateral breast irradiation is usually indicated [3].

Irradiating SBBC with conventional tangential fields has usually challenged with 1) the overlapping treatment fields, 2) difficulties in daily set-up of the patient specially when irradiating regional LNs, 3) increasing the hotspot, and 4) exceeding the tolerance of the dose to organs at risk OAR, making long-term complications inevitable [4].

Herby comes the need to integrate advanced radiotherapy techniques in treating this complex target volume, including intensity-modulated radiation therapy (IMRT) or volumetric-modulated arc therapy (VMAT) [5].

The purpose of this study is to evaluate the role of new radiation techniques (IMRT and VMAT) in solving the dosimetric obstacles in SBBC and comparing them to the conventional 3DCRT.

\section{Material and Methods}

\subsection{Study Design}

Fifteen female patients with pathologically proven synchronous bilateral breast cancer underwent either breast conservative surgery or radical mastectomy for stage I-III disease in whom adjuvant radiotherapy is indicated was selected, any patient with uncontrolled vascular/connective tissue disease, uncontrolled cardiovascular or pulmonary disease, patient who had in-field implanted cardiac devices and patients who received prior chest irradiation was excluded.

Using Varian Eclipse planning system, each patient had three separate plans with VMAT, IMRT and 3DCRT with total dose 50 Gy over 25 fractions to the bilateral breast/ chest wall, with or without supra and infra-clavicular lymph nodes when indicated, no tumor bed boost was allowed, then each plan compared to its counterparts in the terms of PTV coverage ( $D_{\max }$, Dmean, D95\%, D2\%, D105\%, D110\%), and OAR including Lung V20, lung Dmean, cardiac Dmean and V25 and LAD artery Dmean and $\mathrm{D}_{\max }$, also we will compare other parameters including conformity index, homogeneity index and healthy tissue conformity index.

The clinical target volume (CTV) was defined following RTOG contouring atlas, including bilateral breasts and regional LNs when indicated (supraclavicular and axillary LNs), the planning target volume was generated by $5 \mathrm{~mm}$ expan- 
sion all around the CTV with $3 \mathrm{~mm}$ skin trimming [6].

Organs at risk were contoured including the whole lung (both lungs), heart and left anterior descending artery (LAD).

The tolerance doses limit to OAR was following RTOG and QUANTEC, lung $\mathrm{V} 20<30 \%$, V $25<20 \%$, cardiac mean dose $<4$ Gy and for LAD $D_{\max }<30 \mathrm{~Gy}[7]$ [8].

Regarding the 3DCRT, the plan was generated using multi-static field with field in field technique using 8-10 fields depending on SCV field indication, with two isocenter placed at the mid-field on the sternum in case of breasts/chest walls only and placed on the junction between breast/chest wall and SCV in case of SCV irradiation.

IMRT was generated using dynamic method with two isocenters, using 10-12 fields.

VMAT was established using six half arcs ( 3 on each side), with two isocenters.

\subsection{Aim of Study}

Comparing PTV coverage parameters including Dmean, $\mathrm{D}_{\max }, \mathrm{CI}$ and HI, and OAR tolerance doses between the three planning techniques.

\section{Results}

\subsection{Target Volume Coverage and PTV Parameters}

The mean dose delivered to the PTV was $51 \mathrm{~Gy} \pm 0.45$ SD by 3DCRT, $50.32 \mathrm{~Gy}$ $\pm 0.32 \mathrm{SD}$ by IMRT and $51.25 \mathrm{~Gy} \pm 0.7 \mathrm{SD}$ by VMAT with significant differences between the three modalities favoring IMRT $(p=0.001)$, post hoc analysis revealed significant between 3DCRT vs. IMRT $(\mathrm{p}=0.007)$ and significant difference between VMAT and IMRT ( $\mathrm{p}=0.001)$ with no significant difference between 3DCRT and VMAT.

Regarding the dose delivered to 5\% of the PTV (D5\%) there was no significant difference between the three techniques.

The dose delivered to $95 \%$ of the PTV (D95\%) was 47.2 Gy \pm 0.94 SD by 3DCRT, $48.34 \mathrm{~Gy} \pm 0.46 \mathrm{SD}$ by IMRT and $47 \mathrm{~Gy} \pm 0.61$ SD by VMAT with significant difference between the three modalities favoring IMRT $(p=0.001)$, post hoc analysis revealed significant between IMRT vs. 3DCRT $(p=0.004)$ and significant difference between IMRT and VMAT $(\mathrm{p}=0.001)$ with no significant difference between 3DCRT and VMAT.

The maximum dose delivered to the PTV $\left(\mathrm{D}_{\max }\right)$ was $54.7 \mathrm{~Gy} \pm 0.4 \mathrm{SD}$ by 3DCRT, $53.4 \mathrm{~Gy} \pm 0.73 \mathrm{SD}$ by IMRT and $55.7 \mathrm{~Gy} \pm 1$ SD by VMAT with significant difference between the three modalities, more dose delivered with VMAT $(p=0.0001)$, post hoc analysis revealed significant between 3DCRT vs. IMRT ( $p$ $=0.003)$ and significant difference between VMAT and IMRT $(\mathrm{p}=0.0001)$, also with significant difference between VMAT and 3DCRT $(\mathrm{p}=0.02)$.

The volume received $105 \%$ of the prescribed dose (V105\%) was $413.6 \mathrm{cc} \pm 258$ 
SD by 3DCRT, $30 \mathrm{cc} \pm 56 \mathrm{SD}$ with IMRT and $383 \mathrm{cc} \pm 316.7 \mathrm{SD}$ with VMAT with significant difference, more volume receiving it with 3DCRT $(p=0.002)$, this difference was significant when comparing IMRT vs. DCRT and IMRT vs. VMAT, while was non-significant when comparing 3DCRT vs. VMAT.

There was non-significant difference between the three modalities regarding the PTV volume receiving $110 \%$ of the prescribed dose (V110\%).

One of the most important parameters is the conformity index (CI) which significantly differ between the tree treatment techniques favoring IMRT ( $\mathrm{p}=$ 0.0001 ), it was $0.71 \pm 0.12 \mathrm{SD}$ with $3 \mathrm{DCRT}, 0.93 \pm 0.02 \mathrm{SD}$ with IMRT and 0.84 $\pm 0.089 \mathrm{SD}$ with VMAT, there was statistically significant difference when comparing IMRT vs. 3DCRT ( $\mathrm{p}=0.0001$ ), also when comparing VMAT vs. 3DCRT $(\mathrm{p}=0.007)$, with non-significant difference between IMRT and VMAT.

Another important and representative parameter of the whole plan is the homogeneity index (HI), which was $0.88 \pm 0.015$ with 3DCRT, $0.93 \pm 0.014$ by IMRT and $0.88 \pm 0.019$ with VMAT, there was significant difference favoring IMRT ( $\mathrm{p}=$ $0.0001)$, post hoc analysis revealed significant difference between IMRT vs. 3DCRT $(\mathrm{p}=0.0001)$ and between IMRT vs. VMAT $(\mathrm{p}=0.0001)$, with non-significant difference between 3DCRT vs. VMAT (Table 1, Figure 1, Figure 2).

Regarding separately PTVs coverage (Lt breast/chest wall PTV separate and Rt PTV separate) the evaluation parameters including Dmean, D 95\%, D5\%, D $\max$, V105\% and CI, the results for the whole PTV applied with major consistency to the separate PTVs (Table 2).

\subsection{Organs at Risk (OAR) doses and Volumes}

Organs at risk (OAR) were contoured and doses were calculated for lungs, heart and left anterior descending artery (LAD).

For lungs (both lungs) the volume receiving $20 \mathrm{~Gy}$ (V20) was $13.7 \% \pm 2.9 \%$ with 3 DCRT, $17.4 \% \pm 3.2 \%$ by IMRT and $24.8 \% \pm 4.2 \%$ with VMAT with

Table 1. PTV for bilateral breasts.

\begin{tabular}{|c|c|c|c|c|c|c|c|c|c|c|}
\hline \multirow{2}{*}{$\begin{array}{l}\text { Plan Technique } \\
\text { PTV parameters }\end{array}$} & \multicolumn{2}{|c|}{$3 \mathrm{DCRT}^{1}$} & \multicolumn{2}{|c|}{ IMRT $^{2}$} & \multicolumn{2}{|c|}{$\mathrm{VMAT}^{3}$} & \multirow{2}{*}{$P$ value } & \multicolumn{3}{|c|}{$P$ value (post hoc) } \\
\hline & Mean & (SD) & Mean & (SD) & Mean & (SD) & & 1 vs 2 & 1 vs 3 & 2 vs 3 \\
\hline $\mathrm{D}_{5 \%}$ & 5164.10 & 553.766 & 5183.70 & 56.056 & 5352.70 & 92.341 & 0.367 & 0.99 & 0.41 & 0.48 \\
\hline $\mathrm{D}_{95 \%}$ & 4722.30 & 94.080 & 4834.50 & 46.898 & 4705.90 & 61.722 & 0.001 & 0.004 & 0.86 & 0.001 \\
\hline $\mathrm{V}_{105 \%}$ & 413.60 & 258.966 & 30.00 & 56.397 & 383.00 & 316.720 & 0.002 & 0.004 & 0.95 & 0.007 \\
\hline $\mathrm{V}_{110 \%}$ & 0 & 0 & 0 & 0 & 35.40 & 68.943 & 0.09 & NA & NA & NA \\
\hline $\mathrm{D}_{\max }$ & 5474.10 & 40.190 & 5342.00 & 73.562 & 5572.30 & 108.638 & 0.0001 & 0.003 & 0.026 & 0.0001 \\
\hline $\mathrm{CI}$ & 0.7160 & 0.122 & 0.933 & 0.021 & 0.848 & 0.089 & 0.0001 & 0.0001 & 0.007 & 0.099 \\
\hline $\mathrm{HI}$ & 0.88442 & 0.015765 & 0.93291 & 0.014011 & 0.88215 & 0.019834 & 0.0001 & 0.0001 & 0.951 & 0.0001 \\
\hline
\end{tabular}




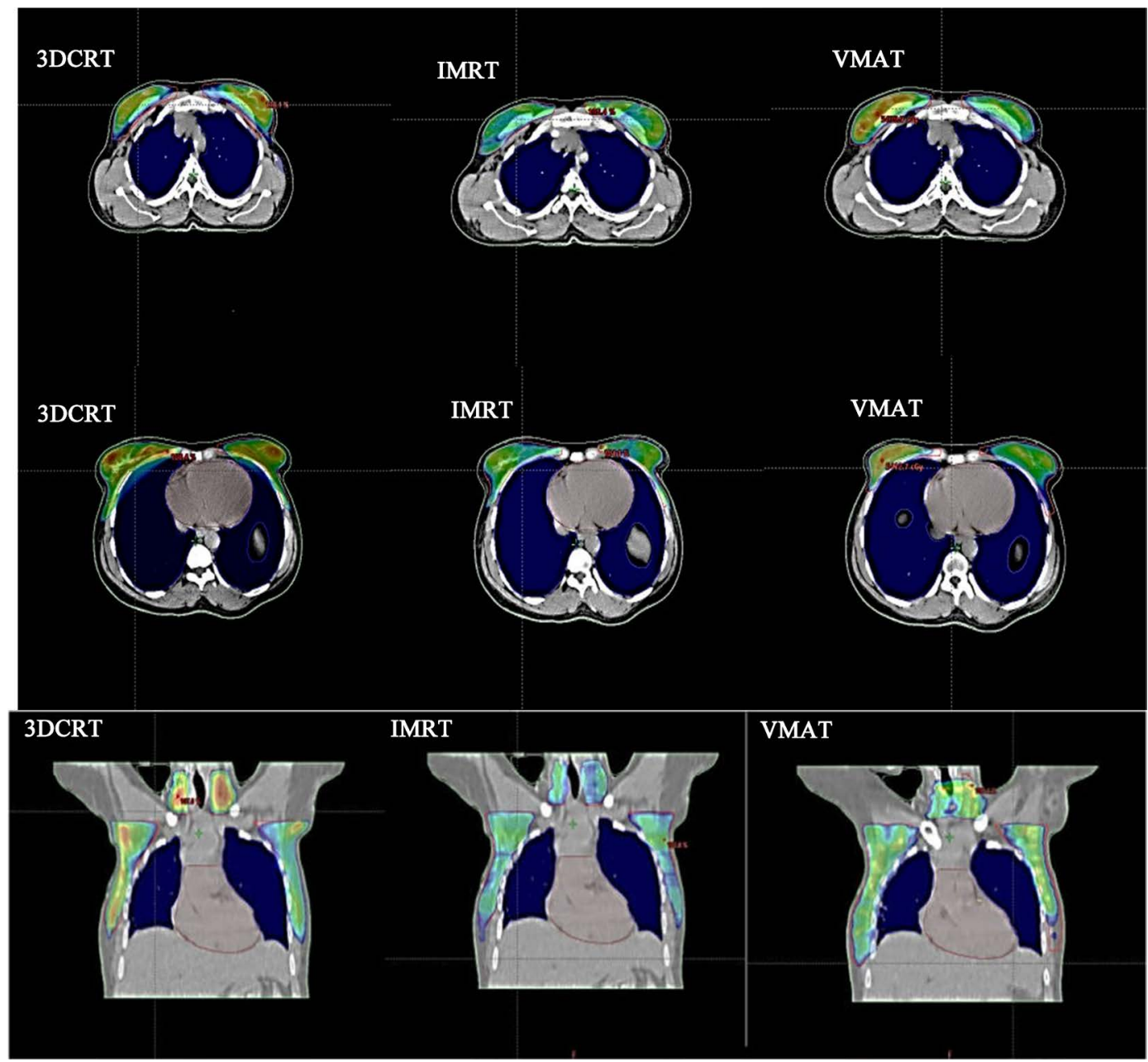

Figure 1. Comparison of D95\% PTV coverage of the three treatment techniques.

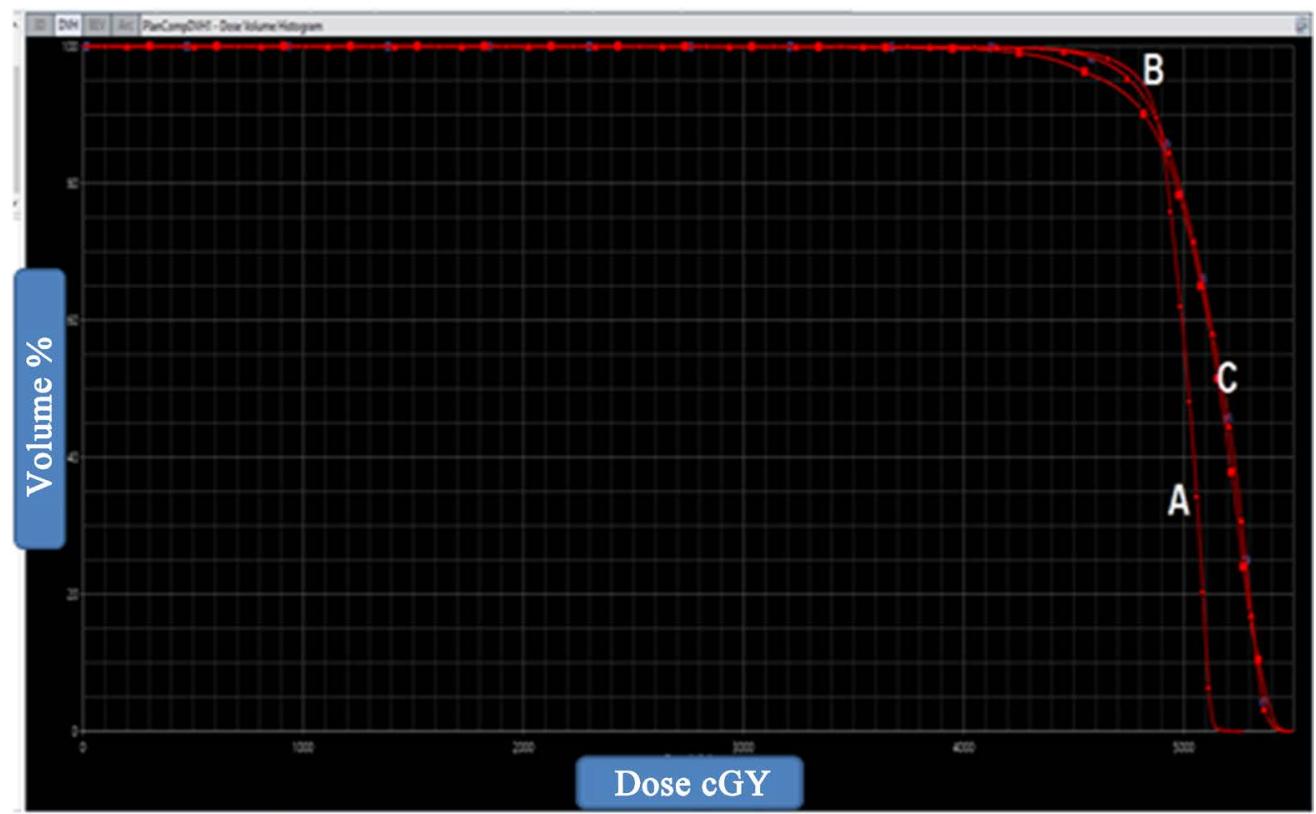

Figure 2. Comparison of dose volume histogram (DVH) of PTV with the 3 techniques (A: 3DCRT, B: IMRT, C: VMAT). 
Table 2. Separate PTVs (Lt/Rt breast).

\begin{tabular}{|c|c|c|c|c|c|c|c|c|c|c|c|}
\hline \multirow{2}{*}{\multicolumn{2}{|c|}{$\begin{array}{l}\text { Plan Technique } \\
\text { PTV parameters }\end{array}$}} & \multicolumn{2}{|c|}{$3 \mathrm{DCRT}^{1}$} & \multicolumn{2}{|c|}{ IMRT $^{2}$} & \multicolumn{2}{|c|}{ VMAT $^{3}$} & \multirow{2}{*}{$P$ value } & \multicolumn{3}{|c|}{$P$ value (post hoc) } \\
\hline & & Mean & (SD) & Mean & (SD) & Mean & (SD) & & 1 vs. 2 & 1 vs. 3 & 2 vs. 3 \\
\hline \multirow{6}{*}{ Left PTV } & $D_{\text {mean }}$ & 5097.50 & 35.522 & 5024.20 & 35.238 & 5091.10 & 67.609 & 0.004 & 0.006 & 0.953 & 0.013 \\
\hline & $\mathrm{D}_{5 \%}$ & 5337.20 & 28.094 & 5179.00 & 54.563 & 5326.70 & 84.149 & 0.0001 & 0.0001 & 0.92 & 0.0001 \\
\hline & $\mathrm{D}_{95 \%}$ & 4720.10 & 75.013 & 4831.00 & 54.829 & 4697.70 & 58.553 & 0.0001 & 0.002 & 0.712 & 0.0001 \\
\hline & $\mathrm{V}_{105 \%}$ & 187.90 & 129.829 & 13.90 & 32.593 & 183.90 & 180.919 & 0.008 & 0.016 & 0.997 & 0.018 \\
\hline & $V_{110 \%}$ & 0 & 0 & 0 & 0 & 12.60 & 32.153 & 0.234 & NA & NA & NA \\
\hline & $\mathrm{D}_{\max }$ & 5468.00 & 36.896 & 5340.89 & 82.575 & 5505.70 & 114.475 & 0.001 & 0.008 & 0.583 & 0.001 \\
\hline \multirow{6}{*}{ Right PTV } & $D_{\text {mean }}$ & 5116.30 & 56.399 & 5032.00 & 32.328 & 5127.80 & 57.813 & 0.0001 & 0.002 & 0.866 & 0.001 \\
\hline & $\mathrm{D}_{5 \%}$ & 5347.40 & 39.331 & 5181.50 & 52.231 & 5366.30 & 93.827 & 0.0001 & 0.0001 & 0.799 & 0.0001 \\
\hline & $\mathrm{D}_{95 \%}$ & 4733.40 & 108.107 & 4838.10 & 42.979 & 4731.90 & 83.522 & 0.01 & 0.023 & 0.999 & 0.021 \\
\hline & $\mathrm{V}_{105 \%}$ & 225.30 & 139.713 & 18.30 & 45.717 & 242.20 & 147.814 & 0.0001 & 0.002 & 0.947 & 0.001 \\
\hline & $V_{110 \%}$ & 0 & 0 & 0 & 0 & 23.40 & 42.220 & 0.063 & NA & NA & NA \\
\hline & $D_{\max }$ & 5469.30 & 43.428 & 5313.90 & 75.368 & 5560.00 & 126.953 & 0.0001 & 0.002 & 0.076 & 0.0001 \\
\hline
\end{tabular}

significant difference favoring 3DCRT ( $\mathrm{p}=0.0001$ ), post hoc analysis revealed non-significant difference between 3DCRT vs. IMRT, but there was significant difference between 3DCRT vs. VMAT ( $\mathrm{p}=0.0001$ ), and between IMRT vs. $\operatorname{VMAT}(\mathrm{p}=0.0001)$.

Also, V10 was significantly lower with 3DCRT $(\mathrm{p}=0.0001)$ post hoc analysis revealed significant difference between 3DCRT vs. IMRT ( $p=0.025)$, and between 3DCRT vs. VMAT ( $\mathrm{p}=0.0001$ ), but no difference between IMRT vs. VMAT.

Again, V5 was significantly lower with 3DCRT ( $\mathrm{p}=0.003)$.

Regarding cardiac doses, the mean dose delivered to the heart was $0.7 \mathrm{~Gy} \pm 1.1$ by 3DCRT, $1.1 \mathrm{~Gy} \pm 1.8$ with IMRT and 0.79 Gy \pm 1.7 with VMAT, with non-significant difference between the three techniques $(\mathrm{p}=0.7)$.

Also, the same applied to V25, which was $2.2 \%$ Gy \pm 2.3 with 3DCRT, $3.1 \% \pm$ $4 \%$ by IMRT and $4 \% \pm 2 \%$ with VMAT, there was non-significant difference between the three modalities.

Regarding LAD, maximum dose delivered was $31.4 \mathrm{~Gy} \pm 1.2$ by 3DCRT, 36.7 Gy \pm 0.9 with IMRT and $38 \mathrm{~Gy} \pm 0.58$ VMAT, with non-significant difference between the three techniques, and the mean dose received by LAD was $13.3 \mathrm{~Gy} \pm$ 0.8 by 3 DCRT, $18 \mathrm{~Gy} \pm 0.9$ with IMRT and $24 \mathrm{~Gy} \pm 0.8$ by VMAT with significant difference favoring the 3DCRT $(\mathrm{p}=0.03)$, post hoc analysis showed non-significant difference between 3DCRT vs. IMRT, and between IMRT vs. VMAT, but there was significant difference between 3DCRT vs. VMAT ( $\mathrm{p}=$ 0.028) (Table 3 and Figure 3).

\section{Discussion}

In our study we tried to find the optimum radiation treatment plan for patients 
Table 3. Organs at risk (OAR).

\begin{tabular}{|c|c|c|c|c|c|c|c|c|c|c|c|}
\hline \multirow{2}{*}{\multicolumn{2}{|c|}{$\begin{array}{l}\text { Plan Technique } \\
\text { PTV parameters }\end{array}$}} & \multicolumn{2}{|c|}{$3 \mathrm{DCRT}^{1}$} & \multicolumn{2}{|c|}{ IMRT $^{2}$} & \multicolumn{2}{|c|}{ VMAT $^{3}$} & \multirow{2}{*}{$P$ value } & \multicolumn{3}{|c|}{$P$ value (post hoc) } \\
\hline & & Mean & (SD) & Mean & (SD) & Mean & (SD) & & 1 vs. 2 & 1 vs. 3 & 2 vs. 3 \\
\hline \multirow{3}{*}{ Whole Lung } & $\mathrm{V}_{20 \mathrm{~Gy}}$ & 13.70 & 2.946 & 17.40 & 3.239 & 24.80 & 4.211 & 0.0001 & 0.065 & 0.0001 & 0.0001 \\
\hline & $\mathrm{V}_{10 \mathrm{~Gy}}$ & 23.10 & 7.680 & 36.40 & 11.037 & 46.90 & 12.609 & 0.0001 & 0.025 & 0.0001 & 0.088 \\
\hline & $\mathrm{V}_{5}$ Gy & 42.90 & 20.306 & 57.10 & 15.279 & 72.30 & 15.326 & 0.003 & 0.172 & 0.002 & 0.136 \\
\hline \multirow{4}{*}{ Heart } & $D_{\text {mean }}$ & 720.20 & 1124.132 & 1139.10 & 1835.844 & 794.80 & 172.870 & 0.728 & 0.735 & 0.990 & 0.812 \\
\hline & $\mathrm{V}_{25 \mathrm{~Gy}}$ & 2.20 & 2.300 & 3.10 & 4.040 & 4.10 & 2.079 & 0.365 & 0.774 & 0.333 & 0.730 \\
\hline & $\mathrm{LAD}_{\max }$ & 3140.20 & 1183.889 & 3671.50 & 909.419 & 3801.70 & 584.137 & 0.256 & 0.416 & 0.264 & 0.947 \\
\hline & $\mathrm{LAD}_{\text {mean }}$ & 1337.10 & 840.982 & 1818.60 & 901.558 & 2404.60 & 863.193 & 0.036 & 0.441 & 0.028 & 0.303 \\
\hline
\end{tabular}

with synchronous bilateral breast cancer, so we compared three radiation techniques including 3DCRT, IMRT and VMAT as regard target volume coverage and OAR dose distribution.

As the target volume in bilateral breast cancer is relatively large specially when combined with reginal LNs irradiation, the standard technique (3DCRT) showed dose inhomogeneity with more area of hot spots and some area of cold spots, so we conducted IMRT and VMAT to find which results in best coverage.

In this study, PTV coverage was best with IMRT compared with 3DCRT and VMAT, this was applied for all the evaluation parameters including mean dose (Dmean), dose delivered to 95\% of the PTV (D95\%), less hotspots including less maximum dose to the PTV $\left(D_{\max }\right)$ and less volume receiving $105 \%$ of the prescribed dose (V105\%).

Also, the most two important parameters which are conformity index (CI) and homogeneity index (HI) was best with IMRT compared with 3DCRT and VMAT.

Regarding OAR dose distribution, there was no difference between the three techniques in cardiac dose, also LAD maximum dose didn't differ significantly, but 3DCRT was best in LAD mean dose and lung volume receiving 20 Gy (V20) and 10 Gy (V10), but the difference was non-significant between 3DCRT and IMRT, while VMAT led to the highest doses to LAD and lung.

Kim SJ, and his colleagues, showed consistent results with our study, they reported that IMRT was the best in conformity index and homogeneity index compared with 3DCRT and IMRT, also improved Dmean and V95\%, in terms of OAR they also concluded that 3DCRT was the best in low dose volume distribution (V20 Gy, V10 Gy, and V5 Gy), for the heart mean dose they showed that 3DCRT was superior but was not statistically significant, also the same results of our study [4].

Cho $\mathrm{Y}$, and his colleagues, conducted a study with different design and different planning techniques for treating SBBC, they compared VMAT with hybrid technique (VMAT foe one PTV and 3DCRT for the other side), but also their results goes with concordance with our results in part that the VMAT is not the 

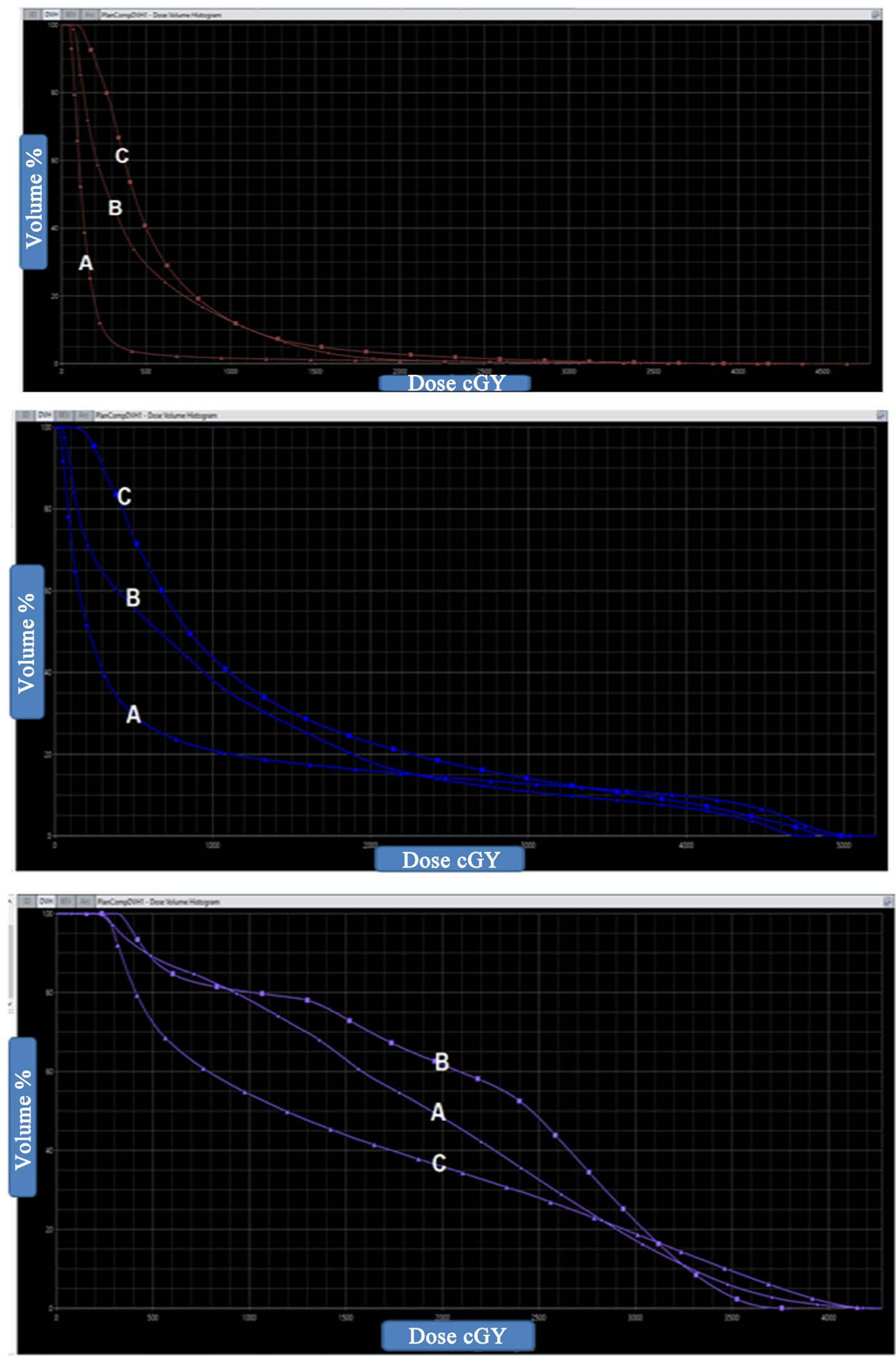

Figure 3. Comparison of dose volume histogram of OAR (1: heart, 2: lung, 3: LAD), A: 3DCRT, B: IMRT, C: VMAT. 
optimum plan, as they concluded that adding 3DCRT to the plan (hybrid technique) is superior to VMAT only plan, and this was consistent in both target volume coverage and OAR doses including lungs V20 Gy and V10 Gy, cardiac and LAD mean doses [1].

Fiorentino $\mathrm{A}$ and his colleagues evaluated the VMAT in adjuvant radiotherapy for SBBC, and they revealed CI $(1.1-1.3)$ compared with 0.84 in our study and this disagreement could be explained as they only calculated the CI for PTV boost only not for the whole PTV as in our study, regarding cardiac dose the mean heart dose was $8.3 \mathrm{~Gy}$ which was similar to mean cardiac dose in our study 7.9 in the VMAT arm, as regard lung V5, V10 and V20 were $79 \%, 39 \%$ and $15.7 \%$ respectively as compared to $72 \%, 47 \%$ and $24.8 \%$ in our study, this slight difference may be due to the use of two arcs instead of three half arcs as in our study [9].

A study by Nicolini and his colleagues, recorded results discordant from our results, as they revealed improved target coverage and OAR dose distribution (heart and lungs), compared with IMRT, this difference could be due to difference in number of fields 2 arcs only in VMAT, field arrangement (fixed field) and more important patients' selection as they include patients with T1 N0 M0, so there was no LNs irradiation and excluding the ribs and inter-costal muscles, so their PTV was smaller than in our study [10].

From all the above-mentioned studies and our study, we saw some agreement in some dosimetric parameters, and disagreement in other parameters, which highlight the need for further research, utilizing different techniques (e.g., combination techniques like IMRT with VMAT in part or 3DCRT in part) and more complexed field arrangement.

\section{Conclusions}

This study was conducted to help in defining the ultimate radiotherapy planning technique for synchronous bilateral breast cancer treatment, and when the three available techniques (3DCRT vs. IMRT vs. VMAT) were compared, we found that IMRT was superior in target volume coverage and this was consistence in all evaluation parameters including mean dose, D95\%, maximum dose, conformity index and homogeneity index.

In terms of organs at risk dose distribution, both 3DCRT and IMRT were superior to VMAT which increased the dose to lung (higher V20\%, V10\%, V5\%) and LAD, due to increment of the volumes receiving low doses with VMAT.

So, our study recommends IMRT as the optimum radiation technique for SBBC treatment, and this is a step toward further research utilizing different techniques to establish solid evidence and recommendations.

\section{Conflicts of Interest}

The authors declare no conflicts of interest regarding the publication of this paper. 


\section{References}

[1] Cho, Y., Cho, Y.J., Chang, W.S., Kim, J.W., Choi, W.H. and Lee, I.J. (2019) Evaluation of Optimal Treatment Planning for Radiotherapy of Synchronous Bilateral Breast Cancer Including Regional Lymph Node Irradiation. Radiation Oncology, 14, 1-8. https://doi.org/10.1186/s13014-019-1257-5

[2] Jobsen, J.J., van der Palen, J., Ong, F., Riemersma, S. and Struikmans, H. (2015) Bilateral Breast Cancer, Synchronous and Metachronous; Differences and Outcome.

Breast Cancer Research and Treatment, 153, 277-283.

https://link.springer.com/article/10.1007/s10549-015-3538-5

https://doi.org/10.1007/s10549-015-3538-5

[3] Kheirelseid, E.A.H., Jumustafa, H., Miller, N., Curran, C., Sweeney, K., Malone, C., et al. (2011) Bilateral Breast Cancer: Analysis of Incidence, Outcome, Survival and Disease Characteristics. Breast Cancer Research and Treatment, 126, 131-140. https://pubmed.ncbi.nlm.nih.gov/20665107/ https://doi.org/10.1007/s10549-010-1057-y

[4] Kim, S.J., Lee, M.J. and Youn, S.M. (2018) Radiation Therapy of Synchronous Bilateral Breast Carcinoma (SBBC) Using Multiple Techniques. Medical Dosimetry, 43, 55-68. https://pubmed.ncbi.nlm.nih.gov/28988893/ https://doi.org/10.1016/j.meddos.2017.08.003

[5] Balaji Subramanian, S., Balaji, K., Thirunavukarasu, M. and Premkumar, S. (2016) Bilateral Breast Irradiation Using Hybrid Volumetric Modulated Arc Therapy (h-VMAT) Technique: A Planning Case Report. Cureus, 8, e914.

https://doi.org/10.7759/cureus.914

[6] White, J., Tai, A., Arthur, D., Buchholz, T., Macdonald, S., Marks, L., et al. (2009) Breast Cancer Atlas for Radiation Therapy Planning: Consensus Definitions Collaborators. Breast Cancer Atlas for Radiation Therapy Planning, 73, 944-951. https://www.rtog.org

[7] Bentzen, S.M., Constine, L.S., Deasy, J.O., Eisbruch, A., Jackson, A., Marks, L.B., et al. (2010) Quantitative Analyses of Normal Tissue Effects in the Clinic (QUANTEC): An Introduction to the Scientific Issues. International Journal of Radiation Oncology, Biology, Physics, 76, S3. https://doi.org/10.1016/j.ijrobp.2009.09.040

[8] Radiation Oncology/Toxicity/RTOG-Wikibooks, Open Books for an Open World [Internet]. https://en.wikibooks.org/wiki/Radiation Oncology/Toxicity/RTOG

[9] Fiorentino, A., Mazzola, R., Naccarato, S., Giaj-Levra, N., Fersino, S., Sicignano, G., et al. (2017) Synchronous Bilateral Breast Cancer Irradiation: Clinical and Dosimetrical Issues Using Volumetric Modulated Arc Therapy and Simultaneous Integrated Boost. Radiologia Medica, 122, 464-471. https://doi.org/10.1007/s11547-017-0741-y

[10] Nicolini, G., Clivio, A., Fogliata, A., Vanetti, E. and Cozzi, L. (2009) Simultaneous Integrated Boost Radiotherapy for Bilateral Breast: A Treatment Planning and Dosimetric Comparison for Volumetric Modulated Arc and Fixed Field Intensity Modulated Therapy. Radiation Oncology, 4, 1-12.

https://doi.org/10.1186/1748-717X-4-27 